Revista del Centro de Investigación de la Universidad La Salle

Vol. 14, No. 56, Julio-Diciembre, 2021: 17-36

DOI: http://doi.org/10.26457/recein.v14i56.2862

\title{
El hologenoma, una herramienta potencial para el estudio de los problemas ambientales ocasionados por xenobióticos
}

\section{The hologenome is a potential tool for the study of environmental problems caused by xenobiotics}

\author{
Rosalba Salgado-Morales \\ Centro de Investigación en Biotecnología (CeIB)-UAEM (México) \\ Edgar Dantán-González \\ Centro de Investigación en Biotecnología (CeIB)-UAEM (México)
}

Recibido: 22 de enero de 2021

Aceptado: 24 de junio de 2021

Publicado: 10 de noviembre de 2021

\section{Resumen}

La presente revisión se centra en el uso potencial del hologenoma como una herramienta para el estudio de problemas ambientales ocasionados por xenobióticos, particularmente de plaguicidas, metales pesados y antibióticos. La revisión aborda una visión general sobre el hologenoma y los avances en los últimos años, así como de las perspectivas y aplicaciones en cuestiones ambientales. Se enfatiza la problemática actual sobre el impacto de los xenobióticos en el ambiente, enfocándonos en el efecto de los plaguicidas sobre organismos no blanco y su microbiota; el impacto de los metales pesados sobre plantas leguminosas y sus bacterias simbiontes y el efecto de los antibióticos principalmente en el sector pecuario. Por último, se analizan algunos organismos modelo para el estudio del hologenoma, entre ellos insectos resistentes a plaguicidas y plantas resistentes a metales pesados desde un contexto de superorganismo u holobionte.

Palabras clave: Hologenoma, Xenobióticos, Plaguicidas, Metales pesados, Antibióticos.

*Email: edantan@uaem.mx

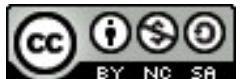

Revista del Centro de Investigación. Universidad La Salle por Dirección de Investigación. Universidad La Salle Ciudad de México se distribuye bajo una Licencia Creative Commons Atribución-NoComercial-CompartirIgual 


\section{Abstract}

This work focuses on the potential use of the hologenome as a tool for the study of environmental problems caused by xenobiotics, particularly pesticides, heavy metals and antibiotics. The review addresses the overview of the hologenome and the advances in recent years, as well as the perspectives and applications in environmental issues. Current issues regarding the impact of xenobiotics on the environment are emphasized, focusing on the effect of pesticides on non-target organisms and their microbiota; the impact of heavy metals on leguminous plants and their symbiotic bacteria and the effect of antibiotics mainly in the livestock activities. Finally, we analyze some model organisms for the study of the hologenome, including insects resistant to pesticides and plants resistant to heavy metals from a superorganism or holobiont context.

Keywords: Hologenome, Xenobiotics, Pesticides, Heavy metals, Antibiotics. 


\section{Introducción}

La influencia de la actividad humana en el antropoceno, es la causa principal del deterioro ambiental a nivel global. La agricultura intensiva, la industrialización, la rápida urbanización y el desarrollo de diversos productos como los xenobióticos, han generado un impacto notable en el ambiente (Lewis y Maslin, 2015). Los plaguicidas utilizados para el manejo de plagas en la agricultura, así como los antibióticos y los metales pesados son algunos de los xenobióticos que se liberan al ambiente, provocando efectos adversos sobre la salud humana y la biota de los ecosistemas. El autor Stephen Palumbi (2001), menciona que la mayor presión evolutiva sobre otras especies es ejercida por las actividades antropogénicas y considera que "los humanos somos la mayor presión evolutiva en del mundo". Sin embargo, la variación genética y la microbiota de los individuos expuestos influye en cómo responde el huésped a la exposición de xenobióticos. En esta revisión abordamos el impacto de los diferentes xenobióticos en el ambiente y destacamos la importancia de la microbiota asociada a los organismos expuestos. Así como el potencial que tiene el hologenoma para contender a la exposición a xenobióticos, desde un contexto de superorganismo u holobionte.

\section{Definiendo el hologenoma}

En la naturaleza las relaciones simbióticas son ubicuas y su impacto ha sido trascendental en la evolución, diversificación de los organismos eucariotas y esencialmente en el éxito de la conquista de diversos ambientes, incluso adversos a los organismos (Angelard y Bever, 2013). La asociación con microorganismos puede ser benéfica, perjudicial o neutral y en algunos casos proveen al hospedero de nuevas capacidades metabólicas. De manera colectiva se define como "microbiota" a los microorganismos que viven en un ambiente definido, aunque en un principio el término se refería a los microorganismos que habitan exclusivamente el intestino humano. Por su parte, el término "microbioma" se refiere a todo el material genético de la microbiota (Lederberg y McCray, 2001; Marchesi y Ravel, 2005). Sin embargo, en años recientes ha tomado suma importancia el estudio de las interacciones entre los hospederos y sus socios microbianos, pero no como unidades autónomas sino como un súper organismo (compuesto por el hospedero más sus socios microbianos) llamado "holobionte". Así la suma de la información genética del hospedero y más la de sus microorganismos simbiontes se denomina "hologenoma" (Zilber-Rosenberg and Rosenberg, 2008; Bordenstein y Theis, 2015; Simon et al., 2019).

\section{Contaminación por xenobióticos}

Los xenobióticos son sustancias químicas extrínsecas al metabolismo del organismo expuesto (Croom, 2012; Spanogiannopoulos et al., 2016). Este término es comúnmente utilizado para referirse a los químicos sintetizados por el hombre, que incluyen muchos químicos recalcitrantes, considerados un problema grave de salud pública y ambiental. También pueden incluir sustancias que se encuentran en la naturaleza, cuya concentración se incrementa por encima de lo usual, incluyendo los derrames industriales o derrames de petróleo (Kanapp y romley-Challoner 2003). Además del origen antrópico, y en un sentido menos estricto, también se consideran xenobióticos a aquellos sintetizados por algunos organismos 
como parte de su sistema de defensa, un ejemplo son las toxinas bacterianas, las micotoxinas y las toxinas producidas por plantas (Soucek, 2011; Abdelsalam et al., 2020). En la actualidad, la exposición a los xenobióticos es prácticamente ineludible y los efectos adversos son ampliamente reconocidos tanto en la salud humana y el ambiente. De manera cotidiana, se da el consumo de alimentos como frutas y vegetales, agua y bebidas como refrescos, vinos y jugos con residuos de xenobióticos. Sin embargo, la exposición a los xenobióticos también puede ser voluntaria, como el caso de los medicamentos y los antibióticos (Nicolopoulou-Stamati et al., 2016). En las últimas décadas, la contaminación por xenobióticos ha incrementado notablemente por la urbanización y las actividades humanas, principalmente en agua y suelo. Las fuentes de contaminación por xenobióticos son diversas e incluyen (I) la industria química y farmacéutica, (II) la industria del blanqueamiento de la pulpa del papel, (III) liberación de residuos de la industria minera como metales pesados, (IV) derrames accidentales de la industria petrolera y (V) la liberación de agroquímicos como plaguicidas y fertilizantes en la agricultura intensiva (Singh 2017; Embrandiri et al., 2017).

\subsection{Plaguicidas}

La agricultura convencional depende en gran medida del uso de fertilizantes y plaguicidas para maximizar la producción y por ende incrementar las ganancias económicas (Aktar y Chowdhury 2009). Después de la Segunda Guerra Mundial, la necesidad de incrementar el rendimiento de los cultivos para cubrir la demanda creciente de alimentos, propició el uso de estos agroquímicos (Popp et al, 2013; Javaid et al, 2016). La Agencia de Protección Ambiental de Estados Unidos (EPA, por sus siglas en inglés) define a los plaguicidas como sustancias o mezclas de sustancias utilizadas para destruir, repeler o mitigar cualquier plaga. Estos productos pueden ser sintetizados químicamente o de origen natural. Se utilizan comúnmente en la agricultura para proteger a los cultivos, principalmente de hierbas e insectos plaga y en el área de la salud pública para el manejo de vectores transmisores de enfermedades como la malaria, fiebre del dengue, chagas, entre otras (Rose, 2001; Blair et al., 2015). Particularmente en la agricultura, las pérdidas por daños causados por plagas son de aproximadamente $45 \%$. Sin embargo, los plaguicidas son la manera más rentable para incrementar el rendimiento de los cultivos hasta en un 30\% a nivel mundial (Bromilow, 2005). El uso de los plaguicidas incrementó notablemente a partir de los años 90s y se estima que 2 millones de toneladas de plaguicidas son utilizados anualmente en todo el mundo. Se estima que para el año 2020 el uso mundial de plaguicidas incremente a 3.5 millones de toneladas anuales (Sharma et al, 2019). Específicamente en Estados Unidos de América, se utilizan anualmente 500 millones de $\mathrm{kg}$ de plaguicidas, con un costo que asciende a los 10 billones de dólares por año (Pimentel et al., 1998; Sharma et al., 2019). En el mercado mundial, esto representa entre el 16 y 18\% del total de plaguicidas, los grupos de plaguicidas mayormente utilizados por EE.UU. son herbicidas (53\%), insecticidas (14\%) y fungicidas (10\%). Por su parte, México es el tercer país con mayor demanda de plaguicidas en América del Norte, después de EE. UU y Canadá. Se estima que para el periodo de 2017-2022, México incremente su demanda aproximadamente 5.2\%. Los principales grupos de plaguicidas utilizados en México son herbicidas e insecticidas que comprende el 36\% del mercado de América del Norte. Uno de los plaguicidas más utilizados entre los años 1997 y 2000 fue el DDT (dicloro difenil tricloroetano), un compuesto organoclorado usado como insecticida principalmente contra vectores de enfermedades como dengue, 
malaria, fiebre amarilla y tifus. Aunque desde 1962 Rachel Carson advertía sobre los efectos adversos de su uso en la Primavera Silenciosa, fue hasta el año 2000 que se prohibió el uso del DDT en México (Sharma et al., 2009; Abhilash y Singh, 2009). Sin embargo, en México y en muchos otros países el uso de otros plaguicidas peligrosos sigue en curso. Entre los principales riesgos del uso de plaguicidas se encuentran: 1). La exposición ocupacional, es decir cuando los trabajadores se encuentran expuestos en su línea de trabajo (agricultores, exterminadores de plagas, jardineros, trabajadores de la industria de los plaguicidas); 2). Exposición de la población (mediante el agua o alimentos contaminados con plaguicidas). Además de los riesgos sobre la salud humana, múltiples evidencias indican que los plaguicidas pueden ocasionar 3). Efectos adversos sobre el ambiente (contaminación del agua, suelo y aire, efectos sobre organismos no blanco: plantas, mamíferos, peces, insectos, etc.) (Damalas y Eleftherohorinos, 2011). Además, el uso repetido del mismo grupo de plaguicida, así como la aplicación de dosis excesivas actúa como una presión de selección sobre la población del organismo plaga, generando cambios en el pool genético e incrementando la población de los individuos resistentes (Kranthi et al., 2002; Naveen et al., 2017). La resistencia a los plaguicidas es definida por el Comité de Acción de Resistencia a Insecticidas (IRAC, por sus siglas en inglés) como un cambio heredable en la sensibilidad de la población de una plaga y se refleja como el fracaso repetido de un producto para lograr el nivel de control esperado cuando se utiliza de acuerdo con las recomendaciones para ese organismo plaga (https://irac-online.org/). Los mecanismos de resistencia a plaguicidas son variados, estos incluyen 1) Resistencia por modificación del sitio blanco: el sitio blanco en donde actúa el plaguicida es modificado y en consecuencia se reduce o elimina el efecto del plaguicida, 2) Resistencia metabólica: se refiere a la capacidad metabólica del organismo para la degradación o detoxificación del plaguicida de manera más rápida que los organismos susceptibles, la actividad catalítica de las enzimas involucradas en metabolizar o desintoxicar está muy relaciona a la resistencia. Entre ellas se encuentran las enzimas citocromo P450, que son un grupo de monooxigenasas que participan en el metabolismo de xenobióticos como: plaguicidas, medicamentos y toxinas vegetales. Estas monooxigenasas, se encuentran en los organismos aerobios como insectos, plantas, mamíferos, aves y también en bacterias. Las monooxigenasas oxidan una amplia variedad de compuestos. Por otro lado, las enzimas Glutation transferasas confieren la resistencia metabólica mediante el secuestro del plaguicida. Es importante mencionar que los simbiontes también son considerados un mecanismo de resistencia, principalmente relacionada a la resistencia metabólica, 3) Resistencia por modificación en la cutícula: la cutícula es una barrera frente a la exposición a los plaguicidas, ciertas modificaciones en la expresión de proteínas cuticulares reducen la penetración del plaguicida al insecto, este tipo de resistencia en conjunto con la resistencia metabólica, pueden conferir altos niveles de resistencia a plaguicidas, 4) Resistencia por transportadores ABC: los transportadores están involucrados en el transporte de moléculas como aminoácidos, azúcares, lípidos, péptidos con dependencia de la hidrólisis de ATP, se encuentran distribuidos en todos los organismos y se ha reportado que juegan un papel importante en la detoxificación de xenobióticos (Figura 1). 
Figura 1

Mecanismos de resistencia a insecticidas

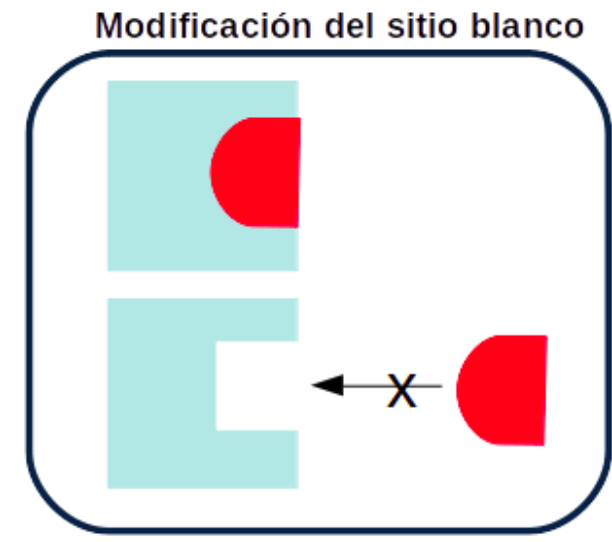

Reducción de la penetración

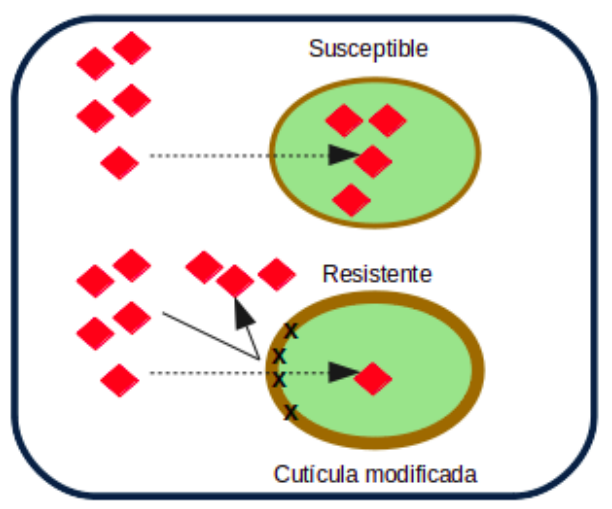

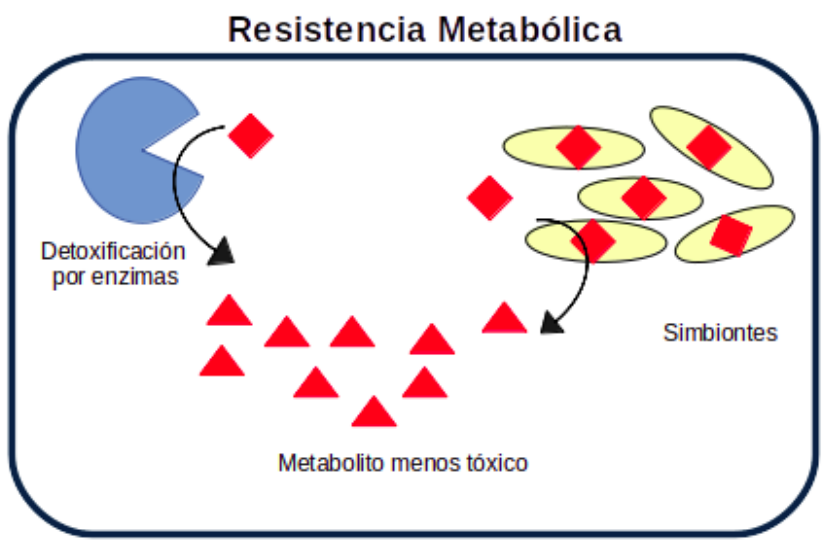

Excreción por transportadores

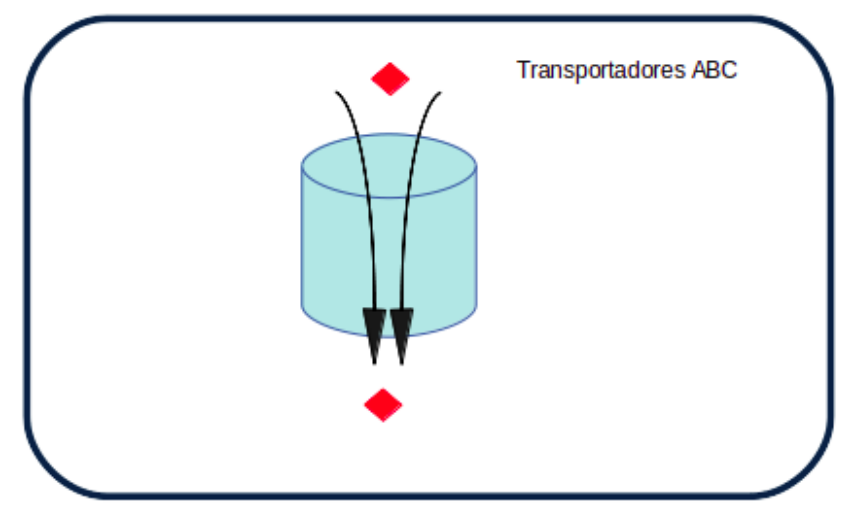

Modificado de Le Goff et al., 2019.

Sin embargo, una amplia diversidad de microorganismos del suelo tienen la capacidad de degradar xenobióticos, incluyendo a los plaguicidas. Estos organismos poseen características genéticas y enzimáticas que les permiten degradar a los plaguicidas en compuestos inorgánicos menos tóxicos (Verma et al., 2014). Las bacterias y los hongos son los principales degradadores. Entre los aislados bacterianos destacan Bacillus, Pseudomonas, Flavobacterium, Arthrobacter, Diaphorobacter, Klebsiella, Ochrobactrum, Agrobacterium, Burkholderia, Kocuria, Archromobacter, Sphingomonas, Chromohalobacter, entre otros que han sido reportados como degradadores de plaguicidas (Ataikiru et al., 2020; Inthama et al., 2021). Las condiciones ambientales, en particular la presencia o ausencia de oxígeno tiene una influencia notable en la degradación de plaguicidas (Reineke, 2001). Los ambientes aeróbicos se caracterizan por la presencia de oxígeno libre $(\mathrm{O} 2)$, en estos ambientes los microorganismos aerobios crecen y llevan a cabo su metabolismo en presencia de oxígeno molecular, realizan la degradación a través de reacciones oxidativas. En contraste, los ambientes anaerobios carecen de oxígeno molecular y los microorganismos anaerobios realizan la degradación a través de reacciones reductoras (Reineke, 2001). 
Los microorganismos pueden utilizar a los plaguicidas como una fuente de nutrientes. Primero el plaguicida entra al microorganismo y posteriormente a través de reacciones enzimáticas lo degradan en moléculas pequeñas no tóxicos o de menor toxicidad (Huang et al., 2018). La capacidad de los microorganismos para la degradación de plaguicidas y su capacidad para adaptarse a ambientes contaminados amplían las capacidades fisiológicas del hospedero y ofrece un gran potencial para contender ante la exposición a plaguicidas (Werren et al 2012; Blanton y Peterson, 2020)

\subsection{Metales pesados $y$ metaloides}

Un grupo importante de xenobióticos son los metales pesados y los metaloides, ambos son elementos inorgánicos. Los metales poseen una gravedad específica cinco veces mayor que el agua, por su parte los metaloides son sustancias intermedias entre metales y no metales, son semiconductores y no presentan lustre, por ejemplo, el arsénico, boro y el silicio (Tchounwou et al., 2012). Ambos se encuentran naturalmente en bajas cantidades en el suelo, en el agua y en la atmósfera, incluso algunos tienen funciones biológicas importantes: cofactores de enzimas y participan en procesos de oxido-reducción. Sin embargo, las actividades antropogénicas contribuyen a incrementar las concentraciones de estos xenobióticos en el ambiente, ocasionando un riesgo para la salud humana y los ecosistemas (He et al., 2005). Los metales y metaloides son los principales xenobióticos que se acumulan en el suelo, derivado de diferentes actividades: son utilizados en el sector agrícola como plaguicidas o fertilizantes, también se utilizan en la industria maderera para protección de la madera, en la industria del vidrio, en la industria petrolera y de manera considerable la industria minera. Esta última genera grandes cantidades de residuos sólidos conocidos como "jales mineros", resultado de la extracción de los minerales (Blowes et al., 2003; Chung et al., 2019). Anualmente se producen 25 mil millones de toneladas de residuos sólidos derivados de las actividades mineras, generando un impacto negativo en los ecosistemas y en la biodiversidad (Mudd y Boger, 2013; Murguía et al., 2016). Los metales pesados resultan ser tóxicos a altas concentraciones para la mayoría de los organismos, en las plantas pueden ocasionar efectos negativos que incluyen una baja en la acumulación de biomasa, clorosis, baja asimilación de nutrientes, desequilibrio hídrico, inhibición del crecimiento y fotosíntesis, senescencia y la muerte (Singh et al., 2016). Sin embargo, algunas han seleccionado mecanismos que les permiten colonizar ambientes con altas concentraciones de metales pesados. En las plantas, la "rizosfera" representa la interfaz entre la planta y la raíz, es el área alrededor de la raíz que se encuentra colonizada por una población específica de microorganismos, resultado de la atracción por los químicos o exudados liberados por la raíz de la planta, el término fue propuesto por el alemán Lorenz Hiltner (1904). Esta asociación con microorganismos puede permitirles contender contra diversos factores ambientales o situaciones de estrés. Por ejemplo, las plantas tolerantes a metales pesados ofrecen en la rizosfera condiciones atractivas para ciertas comunidades microbianas que contribuyen o confieren la tolerancia a los metales pesados, por acumulación, desintoxicación o transformación (Mishra et al., 2017). Además de la capacidad de las plantas para resistir a los metales pesados, los microorganismos como las bacterias endófitas, rizobacterias y hongos han tomado gran interés debido a que incrementan la tolerancia a metales pesados en plantas (Franco-Franklin et al., 2021). El potencial de las plantas tolerantes y microorganismos resistentes a metales pesados ha dado lugar al uso de tecnologías como la fitorremediación, utilizando a la planta y a sus bacterias simbiontes para descontaminar suelos con metales 
pesados (Rajkumar et al., 2012; Kong et al., 2017; Mesa-Marín et al., 2020). Los mecanismos para la captación de los metales pesados incluyen: 1) La bioactivación del metal en la rizosfera por interacciones entre microbios con la raíz de la planta, mediante la alteración de la permeabilidad de la membrana de la planta, cambiando la capacidad de unión de las membranas celulares o por quelación por exudados. 2) Captación del metal por medio de transportadores de metales en la membrana plasmática, incluyen transportadores tipo ATPasa, difusión facilitada de cationes y resistencia natural por la familia de proteínas de macrofagos. 3) Detoxificación de metales por apoplastos, a través de la unión de las paredes celulares y la quelación del metal en el citoplasma por diferentes ligandos como fitoquelatinas, metalotioneínas y proteínas de unión a metales. 4) Secuestración de metales dentro de vacuolas por transportadores localizados en tonoplastos, los metales se concentran en las partes aéreas de la planta en concentraciones superiores a las concentraciones del suelo (Kapagunta, 2017).

\subsection{Antibióticos}

Los antibióticos son otro grupo de xenobióticos, ampliamente utilizados a nivel global como medicamentos para contender contra las infecciones en humanos, animales domésticos y de granja. Los antibióticos son sustancias que inhiben el crecimiento de las bacterias. De manera que matan o evitan que las bacterias se reproduzcan. Los antibióticos son sintetizados naturalmente por diversos organismos o son sintetizados químicamente por el hombre. Además de su uso contra infecciones bacterianas, los antibióticos se utilizan incluso como promotores de crecimiento animal (Gothwal y Shashidhar, 2014). Sin embargo, el uso desmedido de antibióticos y sus descargas en el ambiente ha tenido consecuencias notorias, sobre todo incrementando las poblaciones de bacterias resistentes, impactando en la estructura y diversidad de la microbiota ambiental y generando un serio problema de salud pública (Martínez, 2009; Kraemer et al., 2019). Particularmente en el sector pecuario, el uso de antibióticos es una práctica común, aunado a la creciente demanda y a la producción a gran escala en las granjas, esto podría derivar en un incremento en el consumo de antibióticos hasta en un 67\% para el año 2030 (Van Boeckel et al., 2015). Únicamente en Estados Unidos de América el 80\% de los antibióticos en venta se consumen por el sector pecuario, con el objetivo de prevenir infecciones y mejorar las tasas de crecimiento de los animales para consumo humano. Los antibióticos no son metabolizados completamente en el organismo y son excretados del cuerpo y dispersados en el ambiente, principalmente a través del agua, causando diversos efectos adversos como la contaminación de los ecosistemas y la alteración de diferentes funciones ecológicas como; cambios en la transformación del nitrógeno, metanogénesis, reducción del sulfato, ciclo de nutrientes y alteración en la degradación de la materia orgánica (Roose-Amsaleg y Laverman, 2016; Grenni et al., 2018). La contaminación por antibióticos causa una disminución en la biodiversidad, altera la actividad enzimática y la producción de biomasa (Grenni et al., 2018). Además, el uso de antibióticos con fines no terapéuticos en animales, favorece la resistencia a antibióticos en bacterias patógenas de humanos (Martin et al., 2015). Otro problema importante, es la transmisión de bacterias resistentes por exposición ocupacional, pues hay evidencia de que los entornos antropogénicos como las granjas, influyen sobre el microbioma y resistoma (la colección de genes de resistencia a antibióticos) intestinal de los trabajadores, causando un enriquecimiento de taxas potencialmente patógenos, encontradas también en los ambientes cercanos a las granjas, sugiriendo un intercambio entre el entorno y el personal que labora 
en las granjas (Sun et al., 2020). Estudios indican que, en humanos, la microbiota intestinal está involucrada, entre otros procesos, en la maduración del sistema inmune, modulación de procesos nutricionales y metabólicos (Le Chatelier et al., 2013; Gensollen, et al., 2016; Zheng et al., 2020). La transferencia de la microbiota intestinal humana de gemelos discordantes a ratones axénicos resulta en la transmisión del fenotipo metabólico (obeso o delgado) del humano a los ratones. De manera interesante se observó que la convivencia de ratones obesos y delgados impedía el desarrollo de adiposidad y masa corporal en los ratones obesos, esto se correlacionó con la invasión de Bacteroidales de los ratones delgados a los obsesos y en cierta medida el rescate del fenotipo también se correlacionó con la dieta, indicando que las interacciones de la dieta y la microbiota influencian la biología del hospedero (Ridaura et al., 2013). Los antibióticos también pueden causar la disbiosis de la microbiota y la acumulación de genes de resistencia (Francino, 2015). La resistencia a antibióticos en bacterias ha incrementado de manera notoria y se estima que para el año 2050 las infecciones causadas por patógenos resistentes a antibióticos cobrarán alrededor de 10 millones de vidas al año (O’Neill, 2016). Las bacterias utilizan dos estrategias para poder contender contra los antibióticos: 1) La resistencia mutacional, esto se da a partir del surgimiento de mutaciones que afectan la actividad del fármaco en bacterias que provienen de una población susceptible, generando una modificación del fármaco, reducción de la absorción, activando mecanismos de eflujo para expulsar al fármaco y 2) Adquisición de genes de resistencia por transferencia horizontal, (Munita y Arias, 2016). Entre los ejemplos de mecanismos de modificación del fármaco podemos mencionar modificación de los grupos amino o hidroxilo de aminoglucósidos por enzimas como las adeniltransferasas, acetiltransferasas y fosfotransferasas.

Los antibióticos es probablemente el xenobiótico más estudiado, en cuanto se refiere al efecto de estos sobre las comunidades bacterianas, ya sea por el desequilibrio que ocasiona en los sistemas, sobre todo en la microbiota intestinal o como la resistencia que genera (Greer et al. 2016). Pero también se han descrito la producción de diferentes compuestos antimicrobianos producidos por la microbiota de algunos sistemas (Offret et al. 2016). Lo anterior hace que la relación antibiótico-holobionte, sea un excelente modelo para el estudio y análisis de la evolución, funcionamiento e interacciones del hologenoma.

\section{Discusión}

\section{1. Influencia de la microbiota en la resistencia a xenobióticos}

En el caso de los plaguicidas, existen numerosos reportes sobre la contribución de la microbiota del hospedero en la resistencia de insectos a plaguicidas (Paul y Mandal, 2019). Por ejemplo, Riptortus pedestris mantiene una relación simbiótica con Burkholderia, un simbionte adquirido del ambiente, se aloja en las criptas del intestino del insecto. Este simbionte facilita la degradación del fenitrotion (organofosforado) y contribuye a la resistencia a este insecticida (Kikuchi et al., 2012; Itoh et al., 2018). Por otra parte, Plutella xylostella una plaga importante de crucíferas a nivel mundial, presenta poblaciones resistentes a insecticidas. Además, hay una correlación con un aumento de Firmicutes y menos Proteobacterias en el intestino de las poblaciones resistentes versus la población susceptible. Se ha observado que durante la exposición a los plaguicidas la diversidad de la microbiota intestinal se modifica, específicamente Enterococcus sp. potencia la resistencia a clorpirifos a través de la vitamina $\mathrm{C}$ y ácido acetilsalicílico 
mediante la modulación del sistema inmune (Xia et al., 2013; 2018). En Spodoptera frugiperda también se ha observado que la estructura de la microbiota cambia cuando se exponen a diferentes plaguicidas, la cepa resistente de $S$. frugiperda es un reservorio de bacterias degradadoras de plaguicidas (Almeida et al., 2017). Los casos mencionados anteriormente indican que la microbiota está relacionada con la resistencia a los insecticidas ya sea a través de la modificación de la estructura o por la actividad específica de un simbionte, así como la plasticidad del holobionte para contender contra situaciones de estrés.

Particularmente, las plantas leguminosas se asocian con rizobacterias fijadoras de nitrógeno que proveen varios beneficios a la planta: promoción del crecimiento, productividad, tolerancia al estrés biotico y abiotico, incluyendo la tolerancia a metales pesados. Se ha observado que la leguminosa Crotalaria pumila considerada una planta metalífera, es decir crece en zonas contaminadas con metales pesados, se asocia con Methylobacterium sp., quien es el miembro más dominante del microbioma y responsable de promover la germinación de las semillas, el desarrollo de las plántulas y además se transmite verticalmente como endófito de las semillas aún en condiciones de estrés por metales pesados (Sánchez-López, 2018). Las bacterias resistentes a metales pesados resultan una fuente potencial para el estudio de los genes involucrados en la resistencia. En el caso de $S$. meliloti CCNWSX00200 simbionte de Medicago lupulina, se han identificado mediante análisis transcriptómico y generación de mutantes a seis genes involucrados en la resistencia al $\mathrm{Cu}$ y $\mathrm{Zn}$ : una oxidoreductasa multicobre (CueO), una proteína de la membrana externa (Omp), sulfito oxidoreductasa (YedYZ), además de tres proteínas hipotéticas (una de ellas similar a CusA) (Lu et al., 2017). Los mecanismos para la resistencia de bacterias a metales pesados incluyen cuatro mecanismos particularmente involucrados en la resistencia al $\mathrm{Zn}, \mathrm{Cd} \mathrm{y} \mathrm{Pb}$ : 1) Inmovilización de metales pesados en la pared celular bacteriana por exopolisacáridos (EPS), por ejemplo, en Sinorhizobium meliloti los (EPS) son responsables de la inmovilización de $\mathrm{Cu}^{2+}$ (Hou et al., 2013). 2) Secuestro intracelular de metales pesados por proteínas quelantes, un ejemplo son las proteínas intracelulares SmtA tipo metalotioneínas, las cuales son proteínas ricas en residuos de cisteína que producen sideróforos responsables de la captación de los metales, 3) Secuestro extracelular de metales pesados por precipitación de formas insolubles a través de la excreción de sulfuros y fosfatos que precipitan al Cd y evitan su ingreso a las células, por ejemplo Klebsiella aerogenes y Citrobacter, respectivamente (Macaskie et al., 2000) y 4) Transporte activo de metales pesados por bombas de eflujo, que consisten de proteínas con un dominio transmembrana que transportan iones metálicos del interior como $\mathrm{Cu}+/+2$, $\mathrm{Ag}+, \mathrm{Zn}+2$, al medio extracelular utilizando la hidrólisis del ATP (Lebrazi et al., 2018). En los casos mencionados la relación entre plantas y sus bacterias simbiontes va más allá de la fijación del nitrógeno y promoción de crecimiento, ya que pueden proteger al hospedero de los efectos por la exposición a metales pesados.

\section{Conclusiones}

Los organismos normalmente no aparecen aislados, sino relacionados entre sí con efectos sobre su eficacia biológica. Un holobionte es una forma de organización biológica que resulta de la asociación entre un organismo pluricelular, en el rol de hospedero, junto con su microbioma simbiótico. El microbioma se refiere al conjunto de microorganismos (fundamentalmente bacterias, pero también hongos, virus, etc.) que cohabitan simbióticamente junto con las células del organismo pluricelular (Carrier y Reitzel, 
2017). La evolución en las técnicas de detección de microorganismos durante las dos últimas décadas ha revelado que los organismos pluricelulares conviven con un número elevadísimo de microorganismos simbióticos, en algunos casos (como el humano) tan numeroso como las propias células somáticas (Nolorbe-Payahua, et al. 2020), para tener un panorama más claro de esto último consideremos que el genoma humano contiene alrededor de 20.000 genes, pero su hologenoma contiene $>33$ millones de genes aportados por su microbiota (Lloyd-Price et al., 2016).

Sin lugar a duda el desarrollo de las técnicas moleculares, la bioinformática y las tecnologías NGS ha jugado un papel relevante en la descripción de las comunidades microbianas como habitantes clave de los macroorganismos y como actores en los procesos biológicos, ecológicos y evolutivos; incluso si existen pocos estudios en cuestiones funcionales, en organismos modelo y no modelo y en todos los ecosistemas; ya sean terrestres, marinos o acuáticos, y que abarcan diferentes tipos de asociaciones huésped-microbiota ya sean laxas o estrechas (Bredon et al., 2018).

Se ha establecido por diferentes estudios el camino evolutivo del hologenoma y los holobientes, dónde van cambiando y se van ajustando de acuerdo al paso de la historia evolutiva. A medida que los hábitats cambian debido a las presiones globales y locales, la resiliencia de la población y los procesos de adaptación dependen no solo de sus reservas genéticas, sino también de sus comunidades de bacterias asociadas (Suárez, 2020). Estas relaciones son tan estrechas que incluso se ha desarrollado la teoría evolutiva de unidad de selección, que asegura que el hologenoma resultante de esta organización se ha formado durante millones de años (Morris, 2018), pero también debe mantenerse estable soportando incluso las presiones globales y locales de sus hábitats (Aires y Engelen, 2016).

El progreso reciente en la comprensión de la influencia del hologenoma en la evolución, la ecología y la salud de su huésped, está directamente relacionada con en el desarrollo de herramientas adecuadas para caracterizar y estudiar mejor el proceso de ensamblaje y el funcionamiento de las asociaciones huésped-microbiota. En estudios recientes utilizando la metatranscriptómica se caracterizó la microbiota asociada a las raíces de Holcus lanatus y sus cambios en relación con la composición del suelo. Específicamente, se detectaron cambios en las comunidades de hongos micorrízicos arbusculares de acuerdo con la disponibilidad de fósforo en el suelo (Young et al., 2018).

De forma similar, Cregger y colaboradores (2018) analizan el holobionte de Populus, caracterizan los efectos respectivos de los tejidos y genotipos vegetales sobre la estructura de la comunidad microbiana. Este estudio proporciona una comprensión holística de la estructura del microbioma dentro de una planta hospedante relevante para la bioenergía. Como tal, constituye un atlas o mapa detallado para más pruebas de hipótesis sobre la importancia de taxones microbianos individuales dentro de nichos y hábitats específicos de Populus y una línea de base para comparaciones con otras especies de plantas (Cregger et al., 2018). Lo anterior muestra que el análisis funcional basado en metatranscriptomas es una herramienta poderosa para el estudio de las interacciones naturales entre plantas y microbiomas, que sin lugar a dudas proporciona un potencial enorme en trabajos en control de plagas o en la tolerancia a diversos xenobióticos.

Los hologenomas resultados de estos procesos evolutivos, identificados en todos los sistemas que se han mencionado con anterioridad, adicional a su amplio poder de resiliencia, son potencialmente un 
reservorio casi infinito de genes que pueden funcionar como una sola maquinaria para contender con la presencia de xenobióticos resultantes de las actividades antropogénicas o en algunos casos, como los antibióticos, poder sustituirlos por compuestos que generan menos resistencia y un menor impacto a los sistemas. Como se mencionó, existen diversos ejemplos de mecanismos por los cuales los microorganismos asociados pueden degradar, eliminar, transformar o eludir a estos compuestos.

Otro punto importante, se relaciona con el proceso de ensamblaje de la microbiota y los mecanismos utilizados por el huésped para controlar el reservorio y el mantenimiento de sus socios microbianos. Al alterar experimentalmente la microbiota asociada con Daphnia mediante un tratamiento con antibióticos e inocular a individuos libres de gérmenes con la microbiota alterada, Callens y colaboradores (2018) muestran que la alteración inducida por antibióticos afecta fuertemente al ensamblaje de holobiontes con consecuencias sobre el crecimiento del huésped. Esta alteración provocada por antibióticos tuvo un fuerte efecto en la colonización posterior de Daphnia al afectar las interacciones ecológicas entre los miembros de la microbiota. Demostrando que la composición del grupo de microbiota colonizadora puede ser un factor estructurante importante del ensamblaje de la microbiota en Daphnia, afectando la composición de holobiontes y el crecimiento del hospedador. Estos hallazgos contribuyen a una mejor comprensión de cómo el entorno microbiano puede dar forma a la composición del holobionte y afectar las interacciones huésped-microbiota.

Por otro lado, Vannier y colaboradores (2016) analizan la transmisión de la microbiota asociada a la planta Glechoma hederacea durante la propagación vegetativa. Descubrieron que una proporción significativa de las comunidades microbianas de las plantas madre se transmitían a sus hijas a través de conexiones entre individuos. Lo que ejemplifica la transmisión vertical del hologenoma y su potencial que tiene en la biotecnología aplicada a los cultivos vegetales.

Brener-Raffali y sus colegas (2018) demostraron que tanto los factores genéticos como los ambientales dan forma a la composición microbiana y destacaron en particular la influencia de la temperatura del mar y sus consecuencias sobre la resiliencia de los corales en un contexto de calentamiento global. También informaron del descubrimiento de productos naturales con actividades reguladoras que pueden tener aplicaciones biomédicas potenciales, con lo que se reafirma el reservorio inherente en las comunidades microbianas para el desarrollo de nuevos fármacos.

En estudios similares, Ravanbakhsh y colaboradores (2018) demuestran el papel de la microbiota en la señalización del etileno de plantas, una vía importante en los mecanismos de defensa de las plantas. Guégan y colaboradores (2018) destacan la importancia de la microbiota de insectos en mosquitos, analizan el potencial del holobionte del mosquito para nuevas estrategias para el control de vectores.

Un interesante estudio proporciona nuevos conocimientos sobre la contribución de la microbiota a la digestión de fuentes de alimentos terrestres, lo que puede haber permitido la colonización de la tierra por isópodos terrestres. Se identificaron un repertorio de enzimas complementarias para la degradación de lignocelulosa tanto del huésped como del microbioma en A. vulgare. Lo que proporciona nuevos conocimientos sobre el papel del microbioma en la evolución de los isópodos terrestres y su radiación adaptativa en los hábitats terrestres (Bredon et al., 2018). Este tipo de estudios abre una gran ventana de posibilidades en la búsqueda de enzimas que puedan contribuir en la industria. 
Aunque existen diversos ejemplos en los que utilizan estas maquinarias para resolver problemas ambientales, estos se reducen al uso de genes de manera individual o de algunas bacterias o en el mejor de los casos consorcios bacterianos o mezclas de enzimas, dejando el enorme potencial que representa el TODO. Por lo que resulta imprescindible para el aprovechamiento del hologenoma, el entendimiento de su poder adaptativo, de su funcionamiento y de la complejidad en sus interacciones, para que después puedan ser utilizados como un arsenal en las estrategias para aminorar o reducir el impacto de los xenobióticos.

\section{Agradecimientos}

Se agradece al CONACyT por su apoyo al realizar este trabajo.

\section{Fuentes de financiamiento}

SEP-CONACyT Proyecto: A1-S-22034. Beca otorgada a RSM No. 296934

\section{Disponibilidad de datos}

Los autores declaran que todos los datos utilizados en la investigación estarán disponibles y sin restricciones de acceso.

\section{Referencias}

Abdelsalam, Nehal Adel, Ahmed Tarek Ramadan, Marwa Tarek ElRakaiby, and Ramy Karam Aziz. 2020. "Toxicomicrobiomics: The Human Microbiome vs. Pharmaceutical, Dietary, and Environmental Xenobiotics." Frontiers in Pharmacology, 11, 390. http://doi.org/10.3389/fphar.2020.00390

Abhilash, P C, Sarah Jamil, and Nandita Singh. 2009. "Transgenic Plants for Enhanced Biodegradation and Phytoremediation of Organic Xenobiotics." Biotechnology Advances 27 (4). 474-88. http:// doi.org/10.1016/j.biotechadv.2009.04.002

Aires, Tânia, Ester A Serrão, and Aschwin H Engelen. 2016. "Host and Environmental Specificity in Bacterial Communities Associated to Two Highly Invasive Marine Species (Genus Asparagopsis).” Frontiers in Microbiology 7. 559. http://doi.org/10.3389/fmicb.2016.00559

Aktar, Wasim, Dwaipayan Sengupta, and Ashim Chowdhury. 2009. "Impact of Pesticides Use in Agriculture: Their Benefits and Hazards." Interdisciplinary Toxicology 2 (1). 1-12. http://doi.org/10.2478/ v10102-009-0001-7

Almeida, Luis Gustavo de, Luiz Alberto Beraldo de Moraes, Jose Roberto Trigo, Celso Omoto, and Fernando Luis Consoli. 2017. "The Gut Microbiota of Insecticide-Resistant Insects Houses Insecticide-Degrading Bacteria: A Potential Source for Biotechnological Exploitation.” PloS One 12 (3): e0174754. http://doi.org/10.1371/journal.pone.0174754 
Ataikiru, T. L., Okerentugba, P. O., \& Okpokwasili, G. C. 2020. "Identification of Carbofuran and Paraquat Degrading Microorganisms from Soil." South Asian Journal of Research in Microbiology, 7(1), 40-52. http://doi.org/10.9734/sajrm/2020/v7i130164

Blair, Aaron, Beate Ritz, Catharina Wesseling, and Laura Beane Freeman. 2015. "Pesticides and Human Health.” BMJ Publishing Group Ltd. http://doi.org/10.1136/oemed-2014-102454

Blanton, A. G., \& Peterson, B. F. 2020. "Symbiont-Mediated Insecticide Detoxification as an Emerging Problem in Insect Pests." Frontiers in Microbiology, 11, 2422. http://doi.org/10.3389/fmicb. 2020.547108

Blowes, D W, C J Ptacek, J L Jambor, C G Weisener, D Paktunc, W D Gould, and D B Johnson. 2003. “The Geochemistry of Acid Mine Drainage.” Environmental Geochemistry 9: 149-204. http://doi. org/10.1016/B978-0-08-095975-7.00905-0

Boeckel, Thomas P Van, Charles Brower, Marius Gilbert, Bryan T Grenfell, Simon A Levin, Timothy P Robinson, Aude Teillant, and Ramanan Laxminarayan. 2015. "Global Trends in Antimicrobial Use in Food Animals." Proceedings of the National Academy of Sciences 112 (18): 5649-54. http://doi. org/10.1073/pnas.1503141112

Bredon, Marius, Jessica Dittmer, Cyril Noël, Bouziane Moumen, and Didier Bouchon. 2018. "Lignocellulose Degradation at the Holobiont Level: Teamwork in a Keystone Soil Invertebrate.” Microbiome 6 (1): 1-19. http://doi.org/10.1186/s40168-018-0536-y

Brener-Raffalli K, Clerissi C, Vidal-Dupiol J, Adjeroud M, Bonhomme F, Pratlong M, Aurelle D, Mitta G, Toulza E. 2018. "Thermal regime and host clade, rather than geography, drive Symbiodinium and bacterial assemblages in the scleractinian coral Pocillopora damicornis sensu lato." Microbiome 6 (1): 39. http://doi.org/10.1186/s40168-018-0423-6

Bordenstein, Seth R, and Kevin R Theis. 2015. "Host Biology in Light of the Microbiome: Ten Principles of Holobionts and Hologenomes.” PLoS Biol 13 (8): e1002226. http://doi.org/10.1371/journal. pbio. 1002226

Bromilow, Richard H. 2005. “Assessing the Environmental Behaviour of Pesticides in Agriculture.” In 农药与环境安全国际会议论文集. http://doi.org/10.1016/S0167-8809(96)01096-1

Callens M, Watanabe H, Kato Y, Miura J, Decaestecker E. 2018. . "Microbiota inoculum composition affects holobiont assembly and host growth in Daphnia." Microbiome 6(1):56. http://doi. org/10.1186/s40168-018-0444-1

Carrier, Tyler J, and Adam M Reitzel. 2017. "The Hologenome across Environments and the Implications of a Host-Associated Microbial Repertoire." Frontiers in Microbiology 8: 802. http://doi. org/10.3389/fmicb.2017.00802

Chatelier, Emmanuelle Le, Trine Nielsen, Junjie Qin, Edi Prifti, Falk Hildebrand, Gwen Falony, Mathieu Almeida, Manimozhiyan Arumugam, Jean-Michel Batto, and Sean Kennedy. 2013. "Richness of Human Gut Microbiome Correlates with Metabolic Markers.” Nature 500 (7464): 541-46. http:// doi.org/10.1038/nature12506 
El hologenoma, una herramienta potencial para el estudio de los problemas ambientales ocasionados

por xenobióticos

Chung, Ana Paula, Carina Coimbra, Pedro Farias, Romeu Francisco, Rita Branco, Francisco V Simão, Elsa Gomes, Alcides Pereira, Maria C Vila, and António Fiúza. 2019. “Tailings Microbial Community Profile and Prediction of Its Functionality in Basins of Tungsten Mine." Scientific Reports 9 (1): 1-13. http://doi.org/10.1038/s41598-019-55706-6

Croom, Edward. 2012. "Metabolism of Xenobiotics of Human Environments." In Progress in Molecular Biology and Translational Science, 112:31-88. Elsevier. http://doi.org/10.1186/s40168-018-0413$\underline{8}$

Cregger MA, Veach AM, Yang ZK, Crouch MJ, Vilgalys R, Tuskan GA, Schadt CW. 2018. "The Populus holobiont: dissecting the effects of plant niches and genotype on the microbiome." Microbiome 6 (1): 31. http://doi.org/10.1186/s40168-018-0413-8

Damalas, Christos A, and Ilias G Eleftherohorinos. 2011. "Pesticide Exposure, Safety Issues, and Risk Assessment Indicators." International Journal of Environmental Research and Public Health 8 (5): 1402-19. http://doi.org/10.3390/ijerph8051402

Embrandiri, Asha, S Katheem Kiyasudeen, Parveen Fatemeh Rupani, and Mahammad Hakimi Ibrahim. 2016. "Environmental Xenobiotics and Its Effects on Natural Ecosystem." In Plant Responses to Xenobiotics, 1-18. Springer. http://doi.org/10.1007/978-981-10-2860-1_1

Franco-Franklin, V., Moreno-Riascos, S., \& Ghneim-Herrera, T. (2021). Are endophytic bacteria an option for increasing heavy metal tolerance of plants? A meta-analysis of the effect size. Frontiers in Environmental Science, 8, 294. http://doi.org/10.3389/fenvs.2020.603668

Francino, M P. 2016. "Antibiotics and the Human Gut Microbiome: Dysbioses and Accumulation of Resistances." Frontiers in Microbiology 6: 1543. http://doi.org/10.3389/fmicb.2015.01543

Gensollen, Thomas, and Richard S Blumberg. 2017. "Correlation between Early-Life Regulation of the Immune System by Microbiota and Allergy Development." Journal of Allergy and Clinical Immunology 139 (4): 1084-91. http://doi.org/10.1016/j.jaci.2017.02.011

Gothwal, Ritu, and Thhatikkonda Shashidhar. 2015. "Antibiotic Pollution in the Environment: A Review." Clean-Soil, Air, Water 43 (4): 479-89. http://doi.org/10.3390/microorganisms7060180

Greer R, Dong X, Morgun A, Shulzhenko N. 2016. "Investigating a holobiont: Microbiota perturbations and transkingdom networks." Gut Microbes 7 (2): 126-35. http://doi.org/10.1080/19490976.2015 .1128625

Grenni, P., Ancona, V., \& Caracciolo, A. B. 2018. "Ecological effects of antibiotics on natural ecosystems: A review.” Microchemical Journal, 136, 25-39. http://doi.org/10.1016/j.scitotenv.2018.06.025

Guégan M, Zouache K, Démichel C, Minard G, Tran Van V, Potier P, Mavingui P, Valiente Moro C. 2018."The mosquito holobiont: fresh insight into mosquito-microbiota interactions." Microbiome 6 (1):49. http://doi.org/10.1186/s40168-018-0435-2

He, Zhenli L, Xiaoe E Yang, and Peter J Stoffella. 2005. "Trace Elements in Agroecosystems and Impacts on the Environment." Journal of Trace Elements in Medicine and Biology 19 (2-3): 125-40. http:// doi.org/10.1016/j.jtemb.2005.02.010 
Hou, Wenjie, Zhanqiang Ma, Liangliang Sun, Mengsha Han, Jianjun Lu, Zhenxiu Li, Osama Abdalla Mohamad, and Gehong Wei. 2013. "Extracellular Polymeric Substances from Copper-Tolerance Sinorhizobium Meliloti Immobilize Cu2+.” Journal of Hazardous Materials 261: 614-20. http:// doi.org/10.1016/j.jhazmat.2013.06.043.

Huang, B.; Chen, F.; Shen, Y.; Qian, K.; Wang, Y.; Sun, C.; Zhao, X.; Cui, B.; Gao, F.; Zeng, Z.; Cui, H. 2018. "Advances in Targeted Pesticides with Environmentally Responsive Controlled Release by Nanotechnology." Nanomaterials 8, 102. http://doi.org/10.3390/nano8020102

Inthama, P., Pumas, P., Pathom-Aree, W., Pekkoh, J., \& Pumas, C. 2021. "Plant Growth and Drought Tolerance-Promoting Bacterium for Bioremediation of Paraquat Pesticide Residues in Agriculture Soils." Frontiers in Microbiology, 12, 446. http://doi.org/10.3389/fmicb.2021.604662

Itoh, Hideomi, Kanako Tago, Masahito Hayatsu, and Yoshitomo Kikuchi. 2018. "Detoxifying Symbiosis: Microbe-Mediated Detoxification of Phytotoxins and Pesticides in Insects." Natural Product Reports 35 (5): 434-54. http://doi.org/10.1039/c7np00051k

Javaid, Muhammad Kashif, Mehrban Ashiq, and Muhammad Tahir. 2016. "Potential of Biological Agents in Decontamination of Agricultural Soil.” Scientifica 2016. http://doi.org/10.1155/2016/1598325

Kapagunta, Chandrika 2017. "Role of phytoremediation in treating heavy metal pollution." Knowledge Tank; Project Guru.

Kikuchi, Yoshitomo, Masahito Hayatsu, Takahiro Hosokawa, Atsushi Nagayama, Kanako Tago, and Takema Fukatsu. 2012. "Symbiont-Mediated Insecticide Resistance." Proceedings of the National Academy of Sciences 109 (22): 8618-22. http://doi.org/10.1073/pnas.1200231109

Kong, Z., \& Glick, B. R. 2017. "The role of plant growth-promoting bacteria in metal phytoremediation.” Advances in microbial physiology, 71, 97-132. http://doi.org/10.1016/bs.ampbs.2017.04.001

Knapp, J S, and K C A Bromley-Challoner. 2003. "Recalcitrant Organic Compounds." In Handbook of Water and Wastewater Microbiology, 559-95. Academic Press London. http://doi.org/10.1016/ B978-012470100-7/50035-2

Kraemer, Susanne A, Arthi Ramachandran, and Gabriel G Perron. 2019. "Antibiotic Pollution in the Environment: From Microbial Ecology to Public Policy.” Microorganisms 7 (6): 180. http://doi. org/10.3390/microorganisms 7060180

Lebrazi, Sara, and Kawtar Fikri-Benbrahim. 2018. "Rhizobium-Legume Symbioses: Heavy Metal Effects and Principal Approaches for Bioremediation of Contaminated Soil.” In Legumes for Soil Health and Sustainable Management, 205-33. Springer. http://doi.org/10.1007/978-981-13-0253-4_ 7

Lederberg J, McCray AT. “Ome Sweet 'Omics - a genealogical treasury of words.” Scientist. 2001;15:8.

Le Goff, G., \& Giraudo, M. 2019. "Effects of Pesticides on the Environment and Insecticide Resistance." Olfactory Concepts of Insect Control - Alternative to Insecticides, 51-78. http://doi. org/10.1007/978-3-030-05060-3_3 
El hologenoma, una herramienta potencial para el estudio de los problemas ambientales ocasionados

por xenobióticos

Lewis, S., Maslin, M. 2015. "Defining the Anthropocene.” Nature 519, 171-180. http://doi.org/10.1038/nature14258.

Lloyd-Price, Jason, Galeb Abu-Ali, and Curtis Huttenhower. 2016. "The Healthy Human Microbiome.” Genome Medicine 8 (1): 1-11. http://doi.org/10.1186/s13073-016-0307-y

Lu, Mingmei, Shuo Jiao, Enting Gao, Xiuyong Song, Zhefei Li, Xiuli Hao, Christopher Rensing, and Gehong Wei. 2017. "Transcriptome Response to Heavy Metals in Sinorhizobium Meliloti CCNWSX0020 Reveals New Metal Resistance Determinants That Also Promote Bioremediation by Medicago Lupulina in Metal-Contaminated Soil.” Applied and Environmental Microbiology 83 (20). http://doi.org/10.1128/ AEM.01244-17

Macaskie, Lynne E, Karen M Bonthrone, Ping Yong, and David T Goddard. 2000. "Enzymically Mediated Bioprecipitation of Uranium by a Citrobacter Sp.: A Concerted Role for Exocellular Lipopolysaccharide and Associated Phosphatase in Biomineral Formation." Microbiology 146 (8): 1855-67. http://doi. org/10.1099/00221287-146-8-1855

Marchesi, Julian R, and Jacques Ravel. 2015. "The Vocabulary of Microbiome Research: A Proposal.” Springer. http://doi.org/10.1186/s40168-015-0094-5

Martin, Michael J, Sapna E Thottathil, and Thomas B Newman. 2015. “Antibiotics Overuse in Animal Agriculture: A Call to Action for Health Care Providers." American Public Health Association. http://doi. org/10.2105/AJPH.2015.302870

Martínez, Inés, Grant Wallace, Chaomei Zhang, Ryan Legge, Andrew K Benson, Timothy P Carr, Etsuko N Moriyama, and Jens Walter. 2009. "Diet-Induced Metabolic Improvements in a Hamster Model of Hypercholesterolemia Are Strongly Linked to Alterations of the Gut Microbiota." Applied and Environmental Microbiology 75 (12): 4175-84. http://doi.org/10.1128/AEM.00380-09

Mesa-Marín, J., Pérez-Romero, J. A., Redondo-Gómez, S., Pajuelo, E., Rodríguez-Llorente, I. D., \& Mateos-Naranjo, E. 2020. "Impact of Plant Growth Promoting Bacteria on Salicornia ramosissima Ecophysiology and Heavy Metal Phytoremediation Capacity in Estuarine Soils.” Frontiers in Microbiology, 11, 2148. http://doi.org/10.3389/fmicb.2020.553018

Mishra, Jitendra, Rachna Singh, and Naveen K Arora. 2017. "Alleviation of Heavy Metal Stress in Plants and Remediation of Soil by Rhizosphere Microorganisms." Frontiers in Microbiology 8: 1706. http://doi. org/10.3389/fmicb.2017.01706

Morris, J Jeffrey. 2018. "What Is the Hologenome Concept of Evolution?" F1000Research 7. http://doi. org/10.12688/f1000research.14385.1

Mudd, G M, and D V Boger. 2013. "The Ever Growing Case for Paste and Thickened Tailings-towards More Sustainable Mine Waste Management.” J. Aust. Inst. Min. Metall 2: 56-59.

Munita, Jose M, and Cesar A Arias. 2016. "Mechanisms of Antibiotic Resistance." Virulence Mechanisms of Bacterial Pathogens, 481-511. http://doi.org/10.1128/microbiolspec.VMBF-0016-2015

Murguía, Diego I, Stefan Bringezu, and Rüdiger Schaldach. 2016. "Global Direct Pressures on Biodiversity by Large-Scale Metal Mining: Spatial Distribution and Implications for Conservation.” Journal of Environmental Management 180: 409-20. http://doi.org/10.1016/j.jenvman.2016.05.040 
Nicolopoulou-Stamati, Polyxeni, Sotirios Maipas, Chrysanthi Kotampasi, Panagiotis Stamatis, and Luc Hens. 2016. "Chemical Pesticides and Human Health: The Urgent Need for a New Concept in Agriculture." Frontiers in Public Health 4: 148. http://doi.org/10.3389/fpubh.2016.00148

Nolorbe-Payahua, Christian Deyvis, Anderson Santos de Freitas, Luiz Fernando Wurdig Roesch, and Juliano Zanette. 2020. "Environmental Contamination Alters the Intestinal Microbial Community of the Livebearer Killifish Phalloceros Caudimaculatus.” Heliyon 6 (6): e04190. http://doi.org/10.1016/j.heliyon.2020.e04190

Offret C, Desriac F, Le Chevalier P, Mounier J, Jégou C, Fleury Y. Spotlight on Antimicrobial Metabolites from the Marine Bacteria Pseudoalteromonas: Chemodiversity and Ecological Significance. Mar Drugs. 2016 Jul 8;14(7):129. http://doi.org/10.3390/md14070129.

O’Neill, J. 2019. "Review on Antimicrobial Resistance Antimicrobial Resistance: Tackling a Crisis for the Health and Wealth of Nations. London: Review on Antimicrobial Resistance. 2014."

Palumbi, Stephen, R. 2021. "Humans as the World's Greatest Evolutionary Force ". Science 7;293( 5536):1786-90. http://doi.org/10.1126/science.293.5536.1786

Paul, Debarati, and Santi M Mandal. 2019. "Microbial Adaptation and Resistance to Pesticides." In Bacterial Adaptation to Co-Resistance, 233-49. Springer. http://doi.org/10.1007/978-981-13-8503$\underline{212}$

Pimentel, David, Anthony Greiner, and Tad Bashore. 1998. "Economic and Environmental Costs of Pesticide Use." Environmental Toxicology: Current Developments, 121-50.

Popp, József, Károly Pető, and János Nagy. 2013. "Pesticide Productivity and Food Security. A Review." Agronomy for Sustainable Development 33 (1): 243-55. http://doi.org/10.1007/s13593-012-0105-x

Rajkumar, M., Sandhya, S., Prasad, M. N. V., \& Freitas, H. 2012. Perspectives of plant-associated microbes in heavy metal phytoremediation. Biotechnology advances, 30(6), 1562-1574. http://doi. org/10.1016/j.biotechadv.2012.04.011

Ravanbakhsh M, Sasidharan R, Voesenek LACJ, Kowalchuk GA, Jousset A. 2018. "Microbial modulation of plant ethylene signaling: ecological and evolutionary consequences." Microbiome 6(1): 52. http://doi.org/10.1186/s40168-018-0436-1

Reineke W. 2001. “Aerobic and Anaerobic Biodegradation Potentials of Microorganisms.” In: Beek B. (eds) Biodegradation and Persistence. The Handbook of Environmental Chemistry (Vol. 2 Series: Reactions and Processes), vol 2 / 2K. Springer, Berlin, Heidelberg. http://doi.org/10.1007/10508767_1

Ridaura, Vanessa K, Jeremiah J Faith, Federico E Rey, Jiye Cheng, Alexis E Duncan, Andrew L Kau, Nicholas W Griffin, Vincent Lombard, Bernard Henrissat, and James R Bain. 2013. "Gut Microbiota from Twins Discordant for Obesity Modulate Metabolism in Mice.” Science 341 (6150). http://doi. org/10.1126/science.1241214

Rose, Robert I. 2001. "Pesticides and Public Health: Integrated Methods of Mosquito Management." Emerging Infectious Diseases 7 (1): 17. http://doi.org/10.3201/eid0701.010103 
El hologenoma, una herramienta potencial para el estudio de los problemas ambientales ocasionados

por xenobióticos

Roose-Amsaleg, C., \& Laverman, A. M. 2016. "Do antibiotics have environmental side-effects? Impact of synthetic antibiotics on biogeochemical processes." Environmental Science and Pollution Research, 23(5), 4000-4012. http://doi.org/10.1007/s11356-015-4943-3

Sánchez-López, Ariadna S, Sofie Thijs, Bram Beckers, Ma Carmen González-Chávez, Nele Weyens, Rogelio Carrillo-González, and Jaco Vangronsveld. 2018. "Community Structure and Diversity of Endophytic Bacteria in Seeds of Three Consecutive Generations of Crotalaria Pumila Growing on Metal Mine Residues." Plant and Soil 422 (1-2): 51-66. https://doi.org/10.1007/s11104-0173176-2.

Sharma, Anket, Vinod Kumar, Babar Shahzad, Mohsin Tanveer, Gagan Preet Singh Sidhu, Neha Handa, Sukhmeen Kaur Kohli, Poonam Yadav, Aditi Shreeya Bali, and Ripu Daman Parihar. 2019. "Worldwide Pesticide Usage and Its Impacts on Ecosystem." SN Applied Sciences 1 (11): 1446. http://doi.org/10.1007/s42452-019-1485-1

Sharma, Chhatra Mani, Bjørn Olav Rosseland, Marit Almvik, and Ole Martin Eklo. 2009. "Bioaccumulation of Organochlorine Pollutants in the Fish Community in Lake Årungen, Norway.” Environmental Pollution 157 (8-9): 2452-58. http://doi.org/10.1016/j.envpol.2009.03.007

Simon, Jean-Christophe, Julian R Marchesi, Christophe Mougel, and Marc-André Selosse. 2019. "Host-Microbiota Interactions: From Holobiont Theory to Analysis." Microbiome 7 (1): 1-5. http:// doi.org/10.1186/s40168-019-0619-4

Singh, Samiksha, Parul Parihar, Rachana Singh, Vijay P Singh, and Sheo M Prasad. 2016. "Heavy Metal Tolerance in Plants: Role of Transcriptomics, Proteomics, Metabolomics, and Ionomics." Frontiers in Plant Science 6: 1143. http://doi.org/10.3389/fpls.2015.01143

Soucek, Pavel. 2011. "Xenobiotics BT - Encyclopedia of Cancer.” In, edited by Manfred Schwab, 396467. Berlin, Heidelberg: Springer Berlin Heidelberg. http://doi.org/10.1007/978-3-642-16483$\underline{56276}$

Spanogiannopoulos, Peter, Elizabeth N Bess, Rachel N Carmody, and Peter J Turnbaugh. 2016. "The Microbial Pharmacists within Us: A Metagenomic View of Xenobiotic Metabolism." Nature Reviews Microbiology 14 (5): 273. http://doi.org/10.1038/nrmicro.2016.17

Suárez, Javier, and Adrian Stencel. 2020. “A Part-dependent Account of Biological Individuality: Why Holobionts Are Individuals and Ecosystems Simultaneously.” Biological Reviews. http://doi. $\underline{\operatorname{org} / 10.1111 / \text { brv. } 12610}$

Sun, Jian, Xiao-Ping Liao, Alaric W D’Souza, Manish Boolchandani, Sheng-Hui Li, Ke Cheng, José Luis Martínez, Liang Li, You-Jun Feng, and Liang-Xing Fang. 2020. "Environmental Remodeling of Human Gut Microbiota and Antibiotic Resistome in Livestock Farms." Nature Communications 11 (1): 1-11. http://doi.org/10.1038/s41467-020-15222-y

Tchounwou, Paul B, Clement G Yedjou, Anita K Patlolla, and Dwayne J Sutton. 2012. "Heavy Metal Toxicity and the Environment." In Molecular, Clinical and Environmental Toxicology, 133-64. Springer. http://doi.org/10.1007/978-3-7643-8340-4_6 
Werren, J. H. 2012. "Symbionts provide pesticide detoxification." Proceedings of the National Academy of Sciences, 109(22), 8364-8365. http://doi.org/10.1073/pnas.1206194109

Vannier, N., Bittebiere, AK., Vandenkoornhuyse, P., and Cendrine, M. 2016. "AM fungi patchiness and the clonal growth of Glechoma hederacea in heterogeneous environments." Sci Rep 6, 37852. http://doi.org/10.1038/srep37852

Xia, Xiaofeng, Botong Sun, Geoff M Gurr, Liette Vasseur, Minqian Xue, and Minsheng You. 2018. "Gut Microbiota Mediate Insecticide Resistance in the Diamondback Moth, Plutella Xylostella (L.).” Frontiers in Microbiology 9: 25. http://doi.org/10.3389/fmicb.2018.00025

Xia, Xiaofeng, Dandan Zheng, Huanzi Zhong, Bingcai Qin, Geoff M Gurr, Liette Vasseur, Hailan Lin, Jianlin Bai, Weiyi He, and Minsheng You. 2013. "DNA Sequencing Reveals the Midgut Microbiota of Diamondback Moth, Plutella Xylostella (L.) and a Possible Relationship with Insecticide Resistance." PloS One 8 (7): e68852. http://doi.org/10.1371/journal.pone.0068852

Young, Ellen, Manus Carey, Andrew a Meharg, and Caroline Meharg. 2018. "Microbiome and Ecotypic Adaptation of Holcus Lanatus (L.) to Extremes of Its Soil pH Range, Investigated through Transcriptome Sequencing.” Microbiome 6 (1): 1-26. http://doi.org/10.1186/s40168-018-0434-3

Zheng, Peng, Jing Wu, Hanping Zhang, Seth W Perry, Bangmin Yin, Xunmin Tan, Tingjia Chai, Weiwei Liang, Yu Huang, and Yifan Li. 2020. "The Gut Microbiome Modulates Gut-Brain Axis Glycerophospholipid Metabolism in a Region-Specific Manner in a Nonhuman Primate Model of Depression." Molecular Psychiatry, 1-13. http://doi.org/10.1038/s41380-020-0744-2

Zilber-Rosenberg Zilber-Rosenberg, Ilana, and Eugene Rosenberg. 2008. "Role of Microorganisms in the Evolution of Animals and Plants: The Hologenome Theory of Evolution." FEMS Microbiology Reviews 32 (5): 723-35. http://doi.org/10.1111/j.1574-6976.2008.00123.x 